\title{
Preparation of $\mathrm{WS}_{2}$-PMMA composite films for optical applications
}

Beata M. Szydłowska, ${ }^{1,2}$ Arko Graf, ${ }^{1}$ Adam Kelly, ${ }^{2}$ Werner J. Blau, ${ }^{2}$ Malte C. Gather, ${ }^{3}$ Jana Zaumseil, ${ }^{1,4}$ Claudia Backes ${ }^{1 *}$

${ }^{1}$ Institute for Physical Chemistry, Heidelberg University, D-69120 Heidelberg, Germany

${ }^{2}$ School of Physics and CRANN \& AMBER Research Centres, Trinity College Dublin, Dublin

2, Ireland

3 School of Physics \& Astronomy, University of St Andrews, North Haugh, St Andrews KY169SS, Scotland, UK

${ }^{4}$ Centre for Advanced Materials, Heidelberg University, D-69120 Heidelberg, Germany

* backes@uni-heidelberg.de

\begin{abstract}
Thus far, research activities of 2D materials in optics, photonics and optoelectronics predominantly focus on micromechanically cleaved or grown nanosheets. Here, we show that high quality liquid-exfoliated nanosheets offer an alternative approach. Starting from welldefined, monolayer rich $\mathrm{WS}_{2}$ dispersions obtained after liquid exfoliation and size selection in aqueous surfactant, we present an optimised protocol to transfer the nanosheets to a polymer solution in organic media. From such dispersions, we fabricate $\mathrm{WS}_{2}$-polymer thin films by spin coating. The characteristic photoluminescence of $\mathrm{WS}_{2}$ monolayers is retained in the film at 2.04 $\mathrm{eV}$ without broadening (line width $40 \mathrm{meV}$ ) or significant changes in the line-shape. This confirms that nanosheet aggregation is efficiently prevented on transfer and deposition. The films are extremely smooth and uniform over large areas with a root mean square roughness < $0.5 \mathrm{~nm}$. To demonstrate the potential in optical applications, the nonlinear optical response was studied, revealing promise as optical limiter. In addition, we show that the photoluminescence can be manipulated by coupling the exciton response to cavity photons in a Ag microcavity.
\end{abstract}

\section{Keywords}


Transition Metal Dichalcogenides - Liquid Exfoliation - Monolayer - Composite Films Optical Properties

\section{Introduction}

Layered transition metal dichalcogenides (TMDs) are a diverse source of semiconducting materials interesting for a number of application areas from optoelectronics to energy storage. ${ }^{1-}$

${ }^{9}$ Significant research efforts have been devoted to investigate the unique optical properties of mono- and few-layered nanosheets. ${ }^{10-13}$ Opposed to multilayer or bulk TMDs, monolayers are direct bandgap semiconductors showing reasonable fluorescence quantum yields. ${ }^{10,11}$ As a result of a weak dielectric screening from the environment and thus enhancement of the Coulomb interaction, optical properties of thin-layered TMDs are dominated by strongly bound excitons and trions with large oscillator strength. ${ }^{10,} 11$ As such, these two-dimensional nanosheets are prone to strong light matter coupling and interesting for excitonic device applications, ${ }^{10,12}$ as well as in nonlinear optical applications for example as saturable absorbers or optical power limiters. ${ }^{13}$

While the intriguing optical properties arise from the two-dimensional nature of the nanomaterials, this is at the same time a great challenge for actual applications. For example, while the oscillator strength per atomic unit is large, it is inherently limited because of the single layer character.

For optical applications, the main interest was thus far focused on micromechanically cleaved or grown nanosheets which suffer from inherent limitations such as lack in reliable, large scale fabrication. Recently, a range of new methods were reported, yielding the large-area 2D materials such as thermal decomposition ${ }^{14}$, salt assisted chemical vapour deposition ${ }^{15}$ and metal sulfuration. ${ }^{16}$ Although some of the mentioned methods provide large quantities, these samples still suffer from a problem of inhomogeneity over the large area making implementation of the 2D materials in e.g. photonic devices challenging.

Here, we suggest that nanosheets exfoliated in the liquid phase can provide an alternative approach. Liquid phase exfoliation (LPE) is a widely used production technique to obtain colloidally stable dispersions from various families of layered crystals. ${ }^{17,}{ }^{18}$ A great strength of this method is the ability to prepare composites with a range of other substance classes such as polymers or other nanomaterials which can be useful for a number of applications. ${ }^{18,19}$ For example, graphene and h-BN have been used as fillers in polymer composites to improve 
mechanical strength and/or thermal conductivity or barrier and dielectric properties. ${ }^{20}$ In such applications, nanosheets with lateral dimensions in the $\mu \mathrm{m}$ range, and thickness of $>5-10$ layers are desirable. ${ }^{17-19}$ In contrast, little research has been conducted to explore nanosheet-polymer composites containing predominantly monolayers or few-layers. Such composites could be of interest in optical applications, where the properties of the mono-and few-layered nanosheets are important, as the surrounding polymer matrix can prevent the nanosheets from restacking. This has previously been shown for freeze-dried composites of $\mathrm{WS}_{2}$ which was initially exfoliated in aqueous polyvinyl alcohol solution. ${ }^{20-22}$ However, in this case, no monolayer-rich $\mathrm{WS}_{2}$ dispersions were obtained and the resultant composites were foam-like and thus unsuitable for optical applications, where smooth films are required.

A weakness of liquid exfoliation is that as-produced dispersions have broad nanosheet size and thickness distributions and thus low monolayer contents. Recent research efforts to improve size selection techniques, typically by centrifugation, ${ }^{23}$ have enabled the production of high quality dispersions of monolayer-rich samples. However, such procedures have only been successfully optimised for nanosheets stabilised in aqueous surfactant (or polymer systems). This is not favourable for subsequent film formation. While certain solvents can be used in liquid phase exfoliation, the monolayer yield tends to be lower. ${ }^{24}$

Thus, to explore optical applications of thin films of liquid phase exfoliated nanosheets, it is first required to develop a procedure to prepare dispersions of high monolayer content in organic polymer solution without nanosheet aggregation. Here, we address this challenge and report such a protocol. We use an exfoliation in aqueous surfactant followed by size selection and monolayer enrichment and subsequent transfer of the nanosheets into an organic solvent mixture containing poly(methyl methacrylate) (PMMA) which can be used for the preparation of homogeneous $\mathrm{WS}_{2}-\mathrm{PMMA}$ composite films by spin coating. The characteristic $\mathrm{WS}_{2}$ monolayer photoluminescence is used to optimise the protocol to avoid reaggregation of the nanosheets on transfer and deposition. The narrow band-width A-exciton photoluminescence is maintained in the thin film confirming that monolayer properties are retained due to shielding of the nanosheets from the surrounding polymer. We demonstrate that these films are promising in optical applications by studying their nonlinear optical response and angle-dependent reflectivity after embedding them in a metal microcavity.

\section{Results and Discussion}




\section{Preparation of the dispersions}

To enable the production of $\mathrm{WS}_{2}$-polymer films by spin coating, it is necessary to produce high quality, monolayer-rich dispersions in a polymer solvent mixture that is compatible with the deposition technique. Our general protocol for the preparation of the $\mathrm{WS}_{2}$-PMMA dispersions essentially consists of three main steps: i) exfoliation in aqueous sodium cholate (SC) by probe sonication, ii) size selection by liquid cascade centrifugation and iii) transfer of the $\mathrm{WS}_{2}$ nanosheets from the aqueous surfactant environment to a mixture of PMMA in a tetrahydrofuran (THF) and $N$-methyl-2-pyrrolidone (NMP) solvent blend as detailed in the method section and briefly discussed below.

An aqueous surfactant solution was chosen as stabiliser for the exfoliation, as it has recently been demonstrated that a larger portion of monolayers is obtained with this approach compared to direct exfoliation in solvents. ${ }^{24}$ Size selection was performed to enrich the dispersion in monolayers and to test whether the procedure is applicable to nanosheets of different size and thickness. Therefore, the initial $\mathrm{WS}_{2}$ stock dispersion in $\mathrm{SC}$ was size-selected by liquid cascade centrifugation (LCC). ${ }^{25}$ This technique is an iterative centrifugation, where the stock dispersion is first centrifuged at low centrifugal acceleration $(R C F$, expressed as unit of gravitational force, $g$ ) and the sediment containing unexfoliated material is discarded. The supernatant is subjected to another centrifugation at higher $R C F$. Again, supernatant and sediment are separated and the sediment containing larger/thicker nanosheets collected in $\mathrm{H}_{2} \mathrm{O}$ (no SC to reduce the surfactant concentration), while the supernatant is again centrifuged at higher $R C F$ and the sediment containing smaller/thinner nanosheets is collected in deionised $\mathrm{H}_{2} \mathrm{O}$. In this work, we use centrifugation at $1 \mathrm{~kg}, 5 \mathrm{~kg}$ and $22 \mathrm{~kg}$. As sample nomenclature, both the lower and upper centrifugation boundary of the fraction is indicated: Dispersions containing larger/thicker nanosheets are denoted as $1-5 \mathrm{~kg}$, while the dispersions containing smaller/thinner are denoted as $5-22 \mathrm{~kg}$.

The liquid aqueous medium of the size-selected dispersion was then exchanged to PMMA in organic solvent by a multistep centrifugation procedure. The crucial part of the transfer protocol is that reaggregation of the nanosheets has to be avoided. To achieve an as high as possible loading level of $\mathrm{WS}_{2}$ in the polymer matrix, the $\mathrm{WS}_{2}$ concentration has to be increased simultaneously. This will always be a trade-off because the $\mathrm{WS}_{2}$ in the higher concentration dispersions will be more prone to aggregation. The overall transfer procedure is schematically illustrated in Figure 1 and described in the methods section. The first three centrifugation steps serve to increase the nanosheet concentration and reduce the surfactant content in the 
dispersion. This is essentially a purification/washing procedure to minimise the concentration of surfactant and thus to facilitate the transfer to the organic solvent-based PMMA solution. To achieve this, the $1-5 \mathrm{k} g$ and $5-22 \mathrm{k} g$ dispersions initially obtained from $80 \mathrm{~mL}$ of stock dispersion were centrifuged at $22 \mathrm{~kg}$ and after each step, redispersed in $\mathrm{H}_{2} \mathrm{O}$ with subsequently decreasing the water volume. After these three steps, $1.5 \mathrm{~mL}$ of concentrated $\mathrm{WS}_{2}$ dispersion is obtained which can be directly blended with the polymer-solvent mixture of choice. In this case a 5:2 THF: NMP mixture with a PMMA concentration of $30 \mathrm{~g} / \mathrm{L}$ was chosen, as we found that such dispersions can be used to cast homogeneous films by spin-coating. Note that several modifications of this protocol were tested as discussed in the SI (Figure S1-2).

\section{Characterisation of the dispersions}

To investigate the optical properties of the $\mathrm{WS}_{2}$ nanosheets in aqueous and solvent-based environment, the dispersions were subjected to optical extinction and photoluminescence spectroscopy.

In Figure 2A-B, extinction spectra of small (5-22k $g$, A) and larger (1-5k g, B) $\mathrm{WS}_{2}$ nanosheets are shown before and after transfer to the polymer-solvent medium. No significant change in the spectra is observed for the $5-22 \mathrm{k} g$ before and after transfer, respectively (Figure 2A). However, in the case of the $1-5 \mathrm{k} g$ sample, the spectral profile of the nanosheet in the PMMA solvent mixture is distinct from the dispersion prior to transfer. In particular, the absorbance is superimposed on a background which will be discussed below.

To explain this, it is first important to recall the impact of the nanosheet morphology on the optical spectral. It has been demonstrated that the spectral profile of dispersed $\mathrm{WS}_{2}$ changes with nanosheet size and thickness due to edge and confinement effects. ${ }^{25,26}$ In brief, edge effects are manifested by changes in peak intensity ratios, while the A-exciton position is sensitive to the nanosheet layer number. In aqueous sodium cholate, the spectral profile was previously quantitatively related to the lateral size, $<\mathrm{L}>$, and layer number, $\langle\mathrm{N}>$, respectively. This allows the determination of $<\mathrm{L}>$ and $<\mathrm{N}>$ from the optical spectra. ${ }^{27}$ From the spectra, we calculate nanosheet lateral sizes as $53 \mathrm{~nm}(5-22 \mathrm{~kg}), 68 \mathrm{~nm}(1-5 \mathrm{k} g)$ and mean $<\mathrm{N}>$ as 1.3 and 3.6 layers, respectively. These values were confirmed by atomic force microscopy statistics (Figure 2A-B inset and SI, Figure S3-4).

In addition to changes of the spectral shape due to edge and confinement effects, it was previously shown that extinction spectra (as measured here) can contain a significant contribution from light scattering. ${ }^{26-29}$ By deconvoluting the extinction spectra into absorbance 
and scattering spectra with the aid of an integrating sphere, it was demonstrated that the wavelength-dependent scattering follows a characteristic powerlaw in the non-resonant regime. ${ }^{28}$ In the resonant regime, scattering spectra of transition metal dichalcogenide nanosheets follow the absorbance in shape, albeit with a significant red-shift. ${ }^{25,26}$ The change in the spectral shape of the $\mathrm{WS}_{2}$ in the $1-5 \mathrm{~kg}$ sample after transfer are completely consistent with increased light scattering which strongly suggests that reaggregation occurred.

For LPE WS 2 , a closer analysis of the A-exciton can provide additional information. Due to the empirically found exponential increase in A-exciton energy with decreasing layer number, it is possible to observe a splitting of the A-exciton into contributions from mono-layered (ML) and few-layered (FL) species. ${ }^{25}$ This is useful, since it allows us to potentially track aggregation. To test whether we can distinguish between the mono-layered and few-layered component in the A-exciton, we smoothed the extinction spectra before finding the second derivative with respect to energy, $\mathrm{d}^{2}(\mathrm{Ext}) / \mathrm{dE}^{2}$. Such a procedure is known to narrow contributing peaks roughly threefold, allowing resolution of closely spaced peaks. ${ }^{30}$ Figure $2 \mathrm{C}-\mathrm{D}$ shows second derivatives of the $\mathrm{WS}_{2} \mathrm{~A}$-exciton (1.9-2.1 eV). For the 5-22k $g$ dispersion (Figure 2C), the splitting of the A-exciton resonance is observed. Consistent with previous work, ${ }^{25}$ we find the monolayer peak at $\sim 2.033 \mathrm{eV}$ and the peak attributed to the sum of few layers at $1.993 \mathrm{eV}$ in sodium cholate. After transfer to PMMA-NMP-THF, the ML component is still clearly discernible suggesting negligible aggregation. However, we note that a peak shift to $2.020 \mathrm{eV}$ in the case of the monolayer and $1.985 \mathrm{eV}$ in the case of the few-layer is observed which we attribute to solvatochromism.

In the case of the $1-5 \mathrm{k} g$ sample, hardly any signature from monolayers is observed in the second derivative of the A-exciton in the initial sodium cholate dispersion (Figure 2D). After transfer to PMMA-NMP-THF, the A-exciton is shifted from $1.988 \mathrm{eV}$ to $1.964 \mathrm{eV}$. Interestingly, this shift is larger than in the case of the FL component of the 5-22k $g$ sample. If it was solely due to solvatochromism, this would not be expected. We therefore believe that part of this shift is due to an additional contribution from the red-shifted scattering.

To confirm the high optical quality of the $\mathrm{WS}_{2}$ nanosheets, the samples were investigated by to photoluminescence (PL) spectroscopy. In group VI transition metal dichalcogenides, only monolayers are direct bandgap semiconductors with appreciable PL from the A-exciton. ${ }^{31,32}$ PL can therefore act as a probe sensitive to only monolayered nanosheets. A photoluminescence-excitation (PLE) contour plot of the 5-22k $g$ dispersion in aqueous SC is displayed in Figure 2E. It shows the characteristic emission from the A-exciton at $612 \mathrm{~nm}$ 
(2.026 eV) with a room temperature line width of $\sim 44 \mathrm{meV}$ indicative for a high sample quality. ${ }^{31,33,34}$ The PLE map of the same sample after transfer to PMMA-THF-NMP is shown for comparison in Figure 2F. Unfortunately, the $\mathrm{WS}_{2} \mathrm{~A}$-exciton PL is completely masked by a broad fluorescence background attributed to $\mathrm{NMP}^{35}$ making it impossible to judge the optical quality of the $\mathrm{WS}_{2}$ nanosheets in the solvent-based mixture from PL. Nonetheless, the splitting of the A-exciton resonance in the extinction spectra of the $5-22 \mathrm{k} g$ sample is encouraging, as it suggests that monolayer properties are retained after transfer.

\section{Characterisation of $W_{2}-P M M A$ films}

From the dispersions in PMMA-THF-NMP described above, thin $\mathrm{WS}_{2}$-polymer composite films were prepared by spin coating (see methods). The THF is required to increase the evaporation rate of the organic medium, allow for partial solvent evaporation and thus fabrication of films with sufficient thickness. The $\mathrm{WS}_{2}$-PMMA films were first characterised by extinction spectroscopy (Figure 3A-B, SI Figure S5). The spectral profile is similar to the initial dispersions in sodium cholate confirming the high optical quality of the prepared films. Interestingly, even in the case of the $1-5 \mathrm{k} g$ sample (Figure 3B), no significant scattering background is observed which is in contrast to the dispersion after transfer to PMMA-THFNMP which was used for the spin-coating. This suggests that some heavy mass, large aggregates that can form after transfer to the organic solvent mixture were efficiently pushed off from the substrate by the centripetal acceleration during spin-coating. Films are of yellowgreenish colour with an optical density of $\sim 0.1$ at the A-exciton for the thickest films produced. To further analyse the extinction, the A-exciton regions were smoothed, and $2^{\text {nd }}$ derivatives were calculated (Figure 3A-B insets). The position of the A-exciton is between the values for the aqueous sodium cholate and the PMMA-THF-NMP dispersions. Importantly, the film produced from the 5-22k $g$ dispersion (Figure 3A) shows the characteristic splitting of ML and $\mathrm{FL} \mathrm{WS}_{2}$ in the thin films which implies that the nanosheets are well isolated from each other through the polymer matrix.

To further confirm that the high optical quality of the $\mathrm{WS}_{2}$ nanosheets is retained after formation of the films, Raman spectroscopy was performed with an excitation wavelength of $532 \mathrm{~nm}$ at the lowest possible laser power (see SI) to avoid heating. The resultant spectra for both films compared to the initial dispersions in aqueous sodium cholate are shown in Figure 3E-F. In addition to the dominant $2 \mathrm{LA}(\mathrm{M}) \mathrm{WS}_{2}$ Raman mode at $\sim 354 \mathrm{~cm}^{-1}$, the photoluminescence observed from monolayered $\mathrm{WS}_{2}$ is centred at $\sim 2500 \mathrm{~cm}^{-1}$ (corresponding to $612 \mathrm{~nm}$ ). The ratio of the photoluminescence to Raman can be used as quality indicator of the samples. On the one 
hand, the PL/Raman ratio linearly scales with the volume fraction of monolayer in the sample. ${ }^{25}$ This is reflected in the higher PL/Raman ratio in the 5-22k $g$ dispersion compared to the $1-5 \mathrm{k}$ $g$ dispersion. On the other hand, the PL/Raman ratio in the dispersion compared to the film can be used to trace restacking of the $\mathrm{WS}_{2}$ nanosheets which would reduce the PL/Raman ratio. Importantly, the monolayer PL is only slightly decreased in the films compared to the liquid dispersions for both the 5-22k $g$ sample (Figure 3C) and the 1-5k $g$ sample (Figure 3D), evidencing that only little aggregation occurred during both transfer to the PMMA-THF-NMP mixture and film formation. The PL in the films broadens slightly, but not significantly.

Since the Raman/PL measurements shows that the PL is maintained in thin films, photoluminescence spectra were acquired in a standard fluorescence spectrometer to have convenient access to different excitation wavelengths. Photoluminescence excitation contour plots of the 5-22k $g$ and $1-5 \mathrm{k} g$ films are presented in Figure $3 \mathrm{G}$ and in SI, Figure S6a, respectively. Both PL excitation emission maps were acquired at similar conditions as for the corresponding liquid dispersions of $\mathrm{WS}_{2}$ in aqueous SC. The A-exciton PL features are again clearly visible and well-defined with a similar excitation profile. It is important to note that such measurements are only possible in optically homogeneous films with negligible scattering and material reaggregation proving the high quality of these films.

Furthermore, single emission spectra were extracted from the PLE maps for both liquid aqueous dispersion and film (Figure $3 \mathrm{~h}$ and SI, Figure S6b) and analysed in more detail. They can be fit well with a single Lorentzian suggesting minimal doping from the PMMA matrix in the thin films. The room temperature PL linewidth of the $\mathrm{WS}_{2}$ in the film is slightly narrower than in the dispersion $(40 \mathrm{meV})$ which is indicative for a homogeneous dielectric environment. ${ }^{36}$ Opposed to the PL measurement in the Raman spectrometer, the PL is slightly blue-shifted in the films compared to the initial dispersion. This suggests that the measurement in the Raman spectrometer was affected by heating which is known to broaden and red-shift the PL of $\mathrm{WS}_{2}$ monolayers. ${ }^{37}$ Overall, the photoluminescence measurements demonstrate that the optical properties of the $\mathrm{WS}_{2}$ are preserved in the thin film with nanosheets that are well isolated by the polymer matrix. In addition, the PMMA seems to have a negligible effect on the optical response of $\mathrm{WS}_{2}$.

For optical applications, a low surface roughness is equally important as preventing restacking of the nanosheets in the film. Therefore, AFM was performed on both films (Figure 3C-D). A $10 \times 10 \mu \mathrm{m}$ surface was scanned to calculate the average roughness of the films. As evidenced by the line profiles in the insets of Figure 3C-D, the films are extremely smooth with a root 
mean square (RMS) area roughness of $0.19 \mathrm{~nm}$ and $0.45 \mathrm{~nm}$, respectively which is more than sufficient for many optical applications. To investigate the morphology of the films over larger areas $\left(>10 \mu \mathrm{m}^{2}\right)$ scanning electron microscopy (SEM) was carried out. Representative images are shown in Figure S7 and confirm that the films are very uniform. When mechanically manipulated, the films peel off from the substrate without evidence for rupture. This indicates mechanical robustness and bendability.

\section{Optical application of $W_{2}-P M M A$ films}

\section{Nonlinear optical response (Z-Scan technique)}

2D materials in general and transition metal dichalcogenides in particular possess strong optical nonlinearity and ultrafast response. Proof of principle applications as saturable absorbers in passive mode locking and Q-switching or as optical limiters have been demonstrated. ${ }^{13}$ Here, the energy-dependent nonlinear optical (NLO) response of the $\mathrm{WS}_{2}$ dispersion and the $\mathrm{WS}_{2}$ PMMA thin film was studied in the nanosecond (ns) regime with an open aperture (OA) z-scan system at $532 \mathrm{~nm}$ with the total $z$ distance of $160 \mathrm{~mm}$ with multiple incident energies (see methods). The experimental data collected during measurements for both film and a dispersion were fitted according to the nonlinear beam propagation model (see SI) to extract the nonlinear absorption coefficient $\beta_{\text {eff }}$. Fitted plots of experimental Z-scan spectra are shown in Figure $4 \mathrm{~A} \& \mathrm{~B}$, representative raw data and respective fits are presented in the supporting information (SI, Figure S8).

In the case of the $\mathrm{WS}_{2}$ dispersion, saturable absorption was observed at all incident energies up to $30 \mu \mathrm{J}$ in agreement with literature, with the NLO absorptive coefficient $\beta_{\text {eff }}$ ranging from 40 to $-9 \mathrm{~cm} / \mathrm{GW}$. This is consistent with published $\beta_{\text {eff }}$ values for dispersions of graphene, $\mathrm{MoS}_{2}$, and black phosphorus which are in the range of -70 to $-14 \mathrm{~cm} / \mathrm{GW}$ and reported to occur in the SA regime for similar energy inputs ${ }^{38-41}$. Note that due to different measurements condition and nanosheet morphology, a direct comparison to literature is not meaningful.

In contrast to the dispersion, the film shows a different behaviour: For low incident beam energies $(1 \mu \mathrm{J}, 5 \mu \mathrm{J})$ the film exhibits saturable absorption response with the characteristic upward-facing curves. However, as the energy increases, the peak of the Z-scan trace rises and broadens to finally saturate and flip into downward facing curves (from $7 \mu \mathrm{J}-30 \mu \mathrm{J}$ ). Similar behaviour was previously seen in $\mathrm{MoS}_{2}$-polymer films ${ }^{42}$ and attributed to the transmission 
through the sample being limited most likely by strong excited state absorption (optical limiting).

This clearly demonstrates that the optical limiting (OL) mechanism occurs at much lower energies in a solid film than in a liquid dispersion. In dispersion, nanosheets are expected to first absorb heat from the incident light and once they are saturated, the excess is transferred to the surrounding solvent. In such a system, localized regions of lower refractive index are getting hotter. As a result, the heating creates microbubbles which scatter the incoming light. In contrast, the heat transfer in a film is less efficient, in particular because the material probed is not mobile opposed to a dispersion, where the nanosheets constantly diffuse through the medium. A different nonlinear mechanism is therefore highly probable, resulting from excited charge carriers absorbing more strongly. A heating related effect would result in film damage which appears as asymmetry of the z-scan. This is clearly not observed.

To evaluate the energy-dependent response quantitatively, $\beta$ eff was extracted from the fits and summarised in Table 1, for both dispersion and film.

Table1. Values of nonlinear optical coefficients, $\beta_{\text {eff }}$ for $\mathrm{WS}_{2}-\mathrm{PMMA}$ film and $\mathrm{WS}_{2}-\mathrm{SC}$ dispersion at various laser energies at $532 \mathrm{~nm}$

\begin{tabular}{lllllll}
\hline energy & $1 \mu \mathrm{J}$, & $5 \mu \mathrm{J}$, & $7 \mu \mathrm{J}$, & $15 \mu \mathrm{J}$, & $27 \mu \mathrm{J}$, & \\
\hline Film & -16.47 & -7.20 & 0.62 & 0.79 & 4.38 & $\mathrm{~cm} / \mathrm{GW}$ \\
\hline Dispersion & -9.03 & ------ & -34.43 & -37.34 & -40.46 & $\mathrm{~cm} / \mathrm{GW}$ \\
\hline
\end{tabular}

In Figure $4 \mathrm{C}, \beta_{\text {eff }}$ is plotted as a function of incident energy for visualisation of the results. In both cases (film and the dispersion), the absorptive coefficient changes exponentially and saturates at $\sim 15 \mu \mathrm{J}$ resulting in a plateau at higher energies. For the $\mathrm{WS}_{2}$-PMMA films, the values are negative for energies of 1 and $5 \mu \mathrm{J}$ and positive for 7,15 and $30 \mu \mathrm{J}$, while $\beta_{\text {eff }}$ coefficient for the dispersion is negative across the whole range.

To show the magnitude of the measured nonlinear optical response, the normalized transmission $\Delta T / T_{0}$ is plotted as a function of pulse energy density (fluence) for the $\mathrm{WS}_{2}$ dispersion and PMMA film at different energies (Figure 4D). This not only illustrates that the NLO response in all cases is strongly energy-dependent, but also that it falls into 2 different regimes of saturable absorption and optical limiting character for dispersion and film, respectively. The strength of $\mathrm{OL}$ in the $\mathrm{WS}_{2}$-PMMA film increases rapidly as the energy input exceeds $5 \mu \mathrm{J}$. This demonstrates that the nonlinear optical response of the nanomaterial can be 
conveniently modulated when embedded in thin films. In contrast to dispersions, such films could be used in optical limiting devices.

\section{Control of the light emission via embedding in optical microcavities}

Key characteristics of the linear optical properties of 2D transition metal dichalcogenides arise due to the reduced screening of the excitons which are strongly bound in mono- and few-layered sheets. As such, strong light matter interaction is expected to occur which can be used to manipulate the optical properties of the nanomaterial. ${ }^{12,43}$ This can for example be achieved by coupling of the excitons to cavity photons. Both weak and strong coupling have been achieved with CVD grown or micromechanically-exfoliated monolayer TMDs. ${ }^{12,44-46}$ However, cavity design to maximise the coupling strength is challenging due to the intrinsically limited absorption cross section of the 2D material. From this perspective, LPE nanosheets hold some promise due to the larger available mass of nanomaterial. However, in this case, the challenge of producing high quality thin films in which the monolayer properties are retained has not yet been overcome.

To test whether the films produced in this work are of sufficient quality to enable the investigation of strong light matter coupling, a $200 \mathrm{~nm}$ thick layer ( $\mathrm{WS}_{2}$-PMMA film) was produced by spin-coating and sandwiched between a top $(60 \mathrm{~nm})$ and bottom $(30 \mathrm{~nm})$ semitransparent silver (Ag) mirror. Both reflection spectra and photoluminescence were measured as a function of the in-plane momentum at room temperature in analogy to Graf et $a l .{ }^{47}$ Figure 5A shows the angle-resolved reflectivity of the film. A parabolic cavity mode is observed. The visibility of the mode confirms a homogeneous and smooth layer with very few scattering sites in the film. This demonstrates that the films are in principle of sufficient quality to study light matter interaction. Since no anti-crossing of the parabolic cavity mode with the A-exciton is observed, we conclude that the sample is in the weak light-matter interaction regime. The angleresolved PL map in Figure 5B shows that the emission follows the reflectivity of the cavity mode, i.e. the wavelength of the emission shifts from 600 to $640 \mathrm{~nm}$ depending on the angle. By tuning the cavity thickness, we expect that the emission wavelength of the $\mathrm{WS}_{2}$ nanosheets could be tuned further, thus allowing to customise the optical properties of transition metal dichalcogenide monolayers. This paves the road towards studying light-matter interaction ${ }^{47,48}$ of various 2D nanosheets in optical microcavities in a straightforward manner.

\section{Conclusion}


Thin films of $\mathrm{WS}_{2}$ embedded in a PMMA polymer matrix of high optical quality were fabricated from liquid phase exfoliated $\mathrm{WS}_{2}$ dispersions by spin-coating. Atomic force microscopy showed the films to be extremely smooth with a root mean square area roughness $<0.5 \mathrm{~nm}$. Extinction and photoluminescence measurements of the films confirmed that nanosheet aggregation was prevented by the polymer matrix. The resultant films have an optical density of 0.1 at the A-exciton and the characteristic A-exciton photoluminescence of monolayered nanosheets is maintained. Importantly, PL emission can be well expressed as single Lorentzian with linewidth of $\sim 40 \mathrm{meV}$ at room temperature confirming the high optical quality of films, negligible nanosheets restacking and doping.

To demonstrate potential optical applications, the nonlinear optical response of the film was studied in the nanosecond regime. Here, transition from saturable absorption to optical limiting was observed for an energy input exceeding $5 \mu \mathrm{J}$ demonstrating possibility of nonlinear optical response to be readily modulated in $\mathrm{WS}_{2}$-PMMA composites. Furthermore, the high optical quality of the films was confirmed by angle-resolved reflectivity and photoluminescence after embedding the film in a $\mathrm{Ag}$ microcavity. Formation of a cavity mode across the $\mathrm{WS}_{2} \mathrm{~A}$-exciton and an angle-dependent modulation of the $\mathrm{WS}_{2}$ emission following the cavity mode were observed.

Overall such films are of interest for optical applications and hold a great promise as practical optical limiters for ultrashort laser pulses. The findings above suggests that such $\mathrm{WS}_{2}-\mathrm{PMMA}$ composites are very promising to study light matter interaction of liquid-exfoliated nanosheets. Since such a fabrication procedure can be applied to a range of materials, there is a scope for further fundamental studies on other layered materials.

\section{Methods}

\section{Sample preparation}

$\mathrm{WS}_{2}$ dispersions were prepared by probe sonicating the powder (Sigma Aldrich, order number 243639-50G, initial concentration $30 \mathrm{~g} / \mathrm{L}$ ) in an aqueous surfactant solution (sodium cholate, SC, Sigma Aldrich, order number C1254-100G). The $\mathrm{WS}_{2}$ was immersed in $80 \mathrm{~mL}$ of aqueous surfactant solution (Sodium cholate; $\mathrm{C}_{\mathrm{SC}}=6 \mathrm{~g} / \mathrm{L}$ ) in a $100 \mathrm{~mL}$ metal beaker. The beaker was mounted in a water bath connected to a chiller system to maintain the external temperature at $5^{\circ} \mathrm{C}$. A solid flathead sonication tip was lowered to the bottom of the beaker, then raised 1 $\mathrm{cm}$ above. The mixture was sonicated by probe sonication (Sonics VXC-500) for $1 \mathrm{~h}$ at $60 \%$ 
amplitude with a pulse of $4 \mathrm{~s}$ on and $2 \mathrm{~s}$ off. The dispersion was centrifuged in $20 \mathrm{~mL}$ aliquots using $50 \mathrm{~mL}$ vials in a Hettich Mikro 220R centrifuge equipped with a fixed-angle rotor 1016 at $6 \mathrm{k} \mathrm{rpm}(2660 \mathrm{~g})$ for $1.5 \mathrm{~h}$. The supernatant was discarded. The sediment was collected in 80 $\mathrm{mL}$ of fresh surfactant $\left(\mathrm{C}_{\mathrm{SC}}=2 \mathrm{~g} / \mathrm{L}\right)$ and subjected to a second sonication using the solid flathead tip for $5 \mathrm{~h}$ at $60 \%$ amplitude with a pulse of $4 \mathrm{~s}$ on and $4 \mathrm{~s}$ off. From our experience, this twostep sonication procedure yields a higher concentration of exfoliated $\mathrm{WS}_{2}$ and removes impurities. This dispersion is denoted as stock dispersion.

To select nanosheets by size, liquid cascade centrifugation (Beckman Coulter Avanti XP centrifuge, $15^{\circ} \mathrm{C}$ ) with sequentially increasing rotation speeds was used. Two different rotors were used. For centrifugation at $<5000 \mathrm{~g}$, the JA25.50 fixed angle rotor and $50 \mathrm{~mL}$ centrifuge tubes (VWR, order number 525-0402) were used filled with $20 \mathrm{~mL}$ of dispersion each. For centrifugation at $>5000 \mathrm{~g}$, the JA25.15 rotor was used with $14 \mathrm{~mL}$ vials (Beckman Coulter, order number 331374), filled with $12 \mathrm{~mL}$ dispersion each. The stock dispersion was first transferred into four vials and centrifuged for $2 \mathrm{hrs}$ at $1 \mathrm{k} g$. The sediment containing unexfoliated and very large/thick nanosheets was discarded, and the supernatant transferred into six vials and centrifuged for $2 \mathrm{hrs}$ at $5 \mathrm{k}$. This time sediment was collected in $18 \mathrm{~mL} \mathrm{H}_{2} \mathrm{O}$ and labelled as "1-5k $g \mathrm{WS}_{2}$ ". The supernatant was again transferred equally into six vials and centrifuged $2 \mathrm{hrs}$ at $22 \mathrm{k} g$. The sediment was collected and labelled as " $5-22 \mathrm{k} g \mathrm{WS}_{2}$ " and supernatant discarded.

\section{$\underline{\text { Transfer to organic PMMA solution }}$}

Washing and concentrating of $\mathrm{WS}_{2}$ : The $18 \mathrm{~mL}$ of " $1-5 \mathrm{k} g \mathrm{WS}_{2}$ " and "5-22k $g \mathrm{WS}_{2}$ " were transferred equally into 12 vials $(1.5 \mathrm{~mL}$ Eppendorf centrifuge tubes) and subjected to centrifugation (Hettich Mikro 220R, $15^{\circ} \mathrm{C}, 1195$-A fixed angle rotor) at $22 \mathrm{~kg}$ for $3 \mathrm{~h}$. The sediment was redispersed in $\mathrm{H}_{2} \mathrm{O}$ with reduced volume to a total of 6 (1.5 ml) vials. This sample was again centrifuged at $22 \mathrm{~kg}$ for $3 \mathrm{~h}$ and the sediment collected in $\mathrm{H}_{2} \mathrm{O}$ with reduced volume to a total of $4(1.5 \mathrm{ml})$ vials. This washing was repeated a third time after which the $\mathrm{WS}_{2}$ was redispersed in only $1.5 \mathrm{~mL}$ and referred to as " $\mathrm{WS}_{2}$ concentrate".

Transfer: First, PMMA (350,000 mw) was dissolved in NMP and THF at a concentration of 30 $\mathrm{gL}^{-1}$, respectively, by stirring. The NMP-PMMA and THF-PMMA were mixed at a ratio of 2:5 giving the NMP-THF-PMMA medium. To transfer $\mathrm{WS}_{2}$ into the NMP-THF-PMMA solution, the $\mathrm{WS}_{2}$ concentrate, as well as the NMP-THF-PMMA polymer solution were bath sonicated for $\sim 2$ min. Finally, the $\mathrm{WS}_{2}$ concentrate was injected to the NMP-THF-PMMA at the concentration of choice and gently sonicated to avoid any aggregation. 


\section{Preparation of films}

Glass slides (SCHOTT) with the size of $1.5^{*} 2 \mathrm{~cm}$ were used as substrates for spin coating under argon atmosphere. The $\mathrm{WS}_{2}$-THF-NMP-PMMA dispersions were bath sonicated for 3 minutes to break up aggregates that had eventually formed. To create one $\mathrm{WS}_{2}$-PMMA film a drop (50 $\mu \mathrm{L}$ ) of the dispersion was placed in the middle of the substrate, possibly evenly covering the surface and then spin-coated. To minimise waste of the dispersion a static spin coating with two steps of spinning was chosen. Those were 30s $700 \mathrm{rpms}$ (step 1) and 20s 1000rpm (step 2). The films were left to dry overnight in dehumidified air.

\section{$\underline{\text { Characterisation }}$}

Optical extinction was measured on a Varian Cary $5000 \mathrm{i}$ in $0.5 \mathrm{~nm}$ increments. For dispersions, quartz cuvettes with a pathlength of $0.4 \mathrm{~cm}$ were used.

Atomic force microscopy (AFM) was carried out on a Dimension ICON3 scanning probe microscope (Bruker AXS S.A.S.) in tapping mode in air under ambient conditions using aluminium coated silicon cantilevers (OLTESP-R3). The concentrated dispersions were diluted with water to yield a pale colloidal dispersion. A drop of the dilute $\mathrm{WS}_{2}$-Au dispersions $(20 \mu \mathrm{L})$ was deposited on a pre-heated $\left(180^{\circ} \mathrm{C}\right) \mathrm{Si} / \mathrm{SiO}_{2}$ wafer $\left(1 \times 1 \mathrm{~cm}^{2}\right)$ with an oxide layer of $300 \mathrm{~nm}$. After deposition, the wafer was rinsed with $\sim 5 \mathrm{~mL}$ of water and $\sim 5 \mathrm{~mL}$ of isopropanol. Typical image sizes were $10 \times 10 \mu \mathrm{m}^{2}$ at scan rates of $0.5 \mathrm{~Hz}$ with 1024 lines per image. The $\mathrm{WS}_{2}-\mathrm{PMMA}$ films were measured as obtained without any postprocessing after spin coating.

Raman (Raman/photoluminescence) spectroscopy was performed on the surface of liquid drops of concentrated dispersions using a Renishaw InVia microscope with $532 \mathrm{~nm}$ excitation laser in air under ambient conditions. The Raman emission was collected by a $50 \times$, long working distance objective lens in streamline mode and dispersed by a $24001 / \mathrm{mm}$ grating with $1 \%$ of the laser power $(<0.2 \mathrm{~mW})$. Note that the laser power has to be kept as low as possible to avoid sample decomposition and heating. Measurements on a minimum of five different spots showed the samples to be homogeneous under these measurement conditions. The spectra were baseline corrected and averaged after acquisition. Great care must be taken during the measurement on the surface of liquid drops, as changes in the focal plane during the acquisition will introduce an error in the PL/Raman ratio. $\mathrm{WS}_{2}$-PMMA films were measured as obtained after spin coating with $1 \%$ laser power. In the polymer film, this can still lead to heating effects, but measurement at lower power was not feasible. 
Photoluminescence contour plots were measured using a Horiba Scientific Fluorlog-3 spectrometer equipped with a Xe lamp (450 W) and a PPD-900 photomultiplier tube detector. Measurements were taken under ambient conditions with double monochromators for excitation and emission. Prior to the measurement, the samples were diluted to an optical density of 0.4 (with respect to $1 \mathrm{~cm}$ pathlength) at $430 \mathrm{~nm}$. The quartz cuvettes with $0.4 \times 1 \mathrm{~cm}$ geometry were placed inside the spectrometer so that the excitation light passed through the 0.4 $\mathrm{cm}$ side of the cuvette. Emission was collected in a $90^{\circ}$ angle, i.e. after having passed through the $1 \mathrm{~cm}$ side. Bandwidths were $5 \mathrm{~nm}$ with acquisition times of $0.4 \mathrm{~s}$. To avoid artefacts from scattering of the nanomaterial dispersion, a $550 \mathrm{~nm}$ cut-off filter was placed on the emission side. The excitation was corrected for the light intensity.

The open-aperture Z-scan ${ }^{41}$ was used to measure the total transmittance of samples as a function of incident intensity. An $\mathrm{f}=30 \mathrm{~cm}$ lens is used to vary the beam diameter and hence the intensity as the sample travels through its focus (in z-direction). All z-scan experiments were performed with 6 ns pulses from a Q-switched Nd:YAG laser. The beam was spatially filtered to remove higher-order modes and tightly focused for all experiments. The laser was operated as second harmonic at $532 \mathrm{~nm}$ with a pulse repetition rate of $10 \mathrm{~Hz}$. The linear transmission of the 5-22k $g$ film was estimated to be $\sim 30 \%$ at $532 \mathrm{~nm}$. Open aperture (OA) z-scans were performed with multiple incident energies set at $1 \mu \mathrm{J}, 5 \mu \mathrm{J}, 7 \mu \mathrm{J}, 15 \mu \mathrm{J}$ and $30 \mu \mathrm{J}$. Each measurement has been repeated 20 times to eliminate random errors, possibly exclude outliers and finally averaged to get reliable information on the NL response of the examined samples. As a reference, the waterbased dispersion in quartz cuvettes (1 mm pathlength) was also characterised with the same technique at identical measurements conditions with the linear transmission through the dispersion at $532 \mathrm{~nm}$ wavelength fixed to $20 \%$. The OA z-scans were performed with multiple incident energies set at $1 \mu \mathrm{J}, 7.5 \mu \mathrm{J}, 15 \mu \mathrm{J}$ and $30 \mu \mathrm{J}, 20$ times each.

Angle-dependent reflectivity and photoluminescence in the microcavity: A $60 \mathrm{~nm}$ thick $\mathrm{Ag}$ mirror was thermally evaporated on a polished Si wafer. Thin polymer films containing $\mathrm{WS}_{2}$ (200 nm thickness) were spin-coated onto this mirror as outlined above. The cavity was finalised by evaporating a $30 \mathrm{~nm}$ Ag mirror on top. The $\mathrm{WS}_{2}$-PMMA film was investigated on a custom-built inverted fluorescence microscope. The excitation pulses were produced by an optical parametric oscillator (OPO; Opolette 355, Opotek Inc.) tuned to $425 \mathrm{~nm}$ (repetition rate, $\max 20 \mathrm{~Hz}$; pulse duration, $5 \mathrm{~ns}$ ). The spectra were recorded with an EM-CCD camera (Newton 971, Andor) coupled to a spectrograph (Shamrock 500i, Andor). 



\section{Figures}

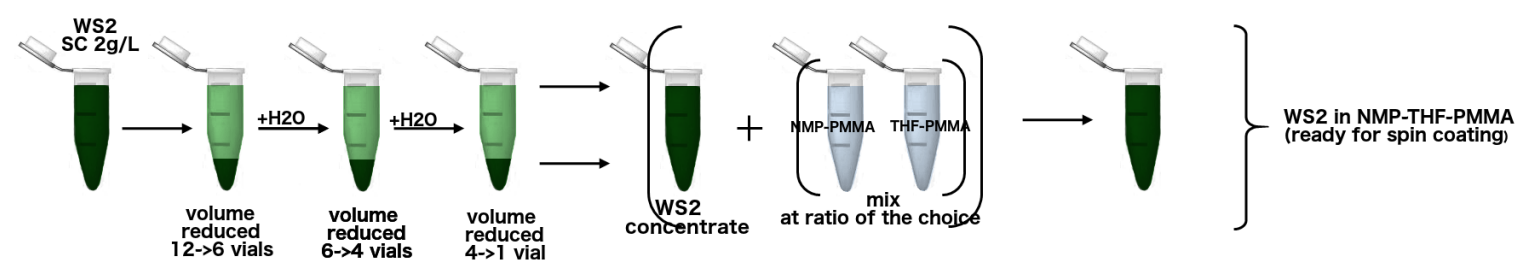

Figure 1: Schematic of the transfer procedure to yield $W S_{2}$ in PMMA-THF-NMP after exfoliation and size selection in aqueous sodium cholate (SC). The dispersion in aqueous SC was first centrifuged multiple times and redispersed in water to remove SC and yield concentrated $W S_{2}$ dispersions which can be directly mixed with PMMA-THF-NMP for further processing by spin coating. 

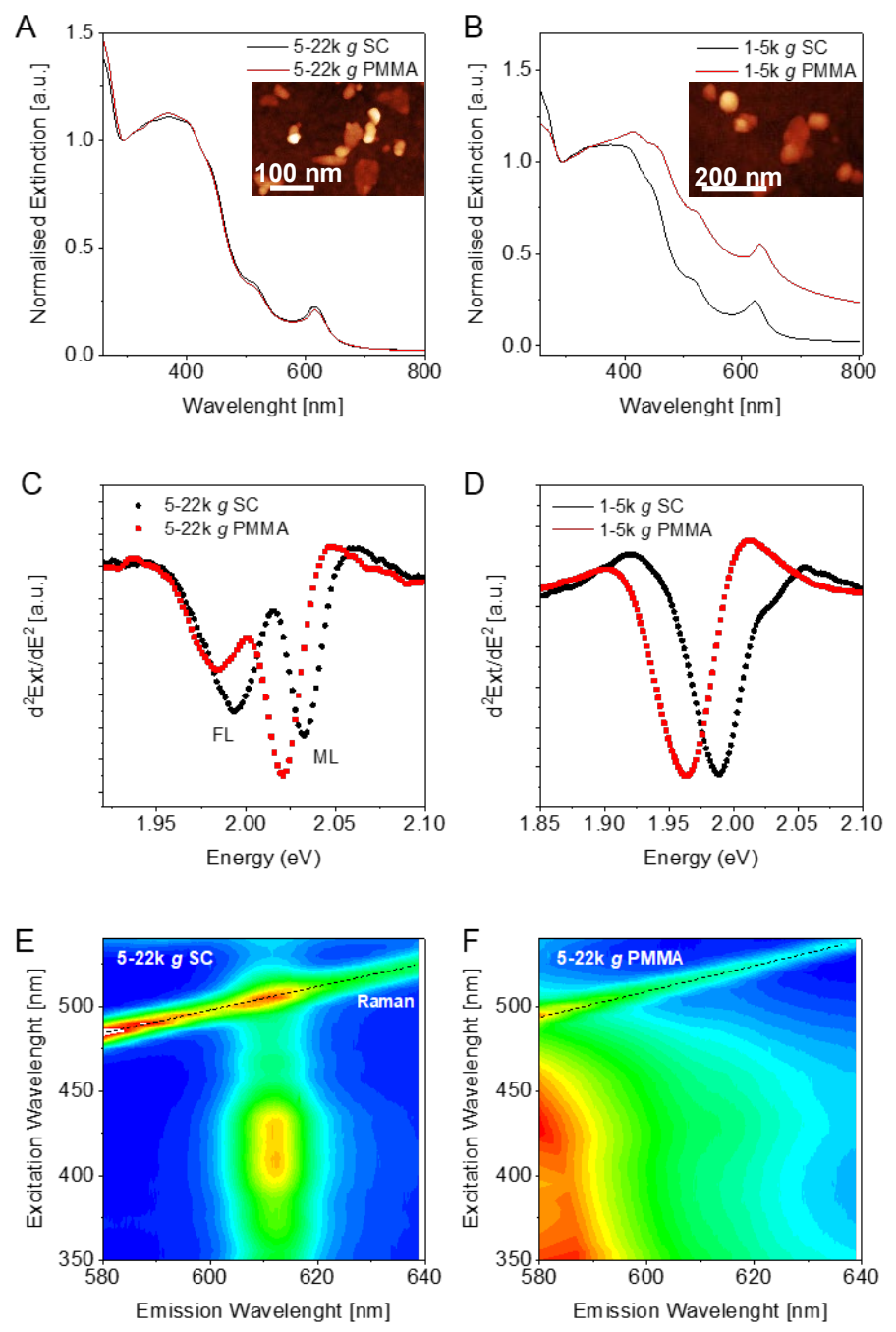

Figure 2: Characterisation of the WS 2 dispersions. A-B) Extinction spectra of small $(5-22 \mathrm{~kg}$, A) and larger (1-5k g, B) WS in aqueous sodium cholate (SC) and after transfer to PMMATHF-NMP. Inset: representative AFM images of the $W S_{2}$ nanosheets. $\left.C-D\right)$ Second derivative of the A-exciton of small $W S_{2}(5-22 \mathrm{~kg}, C)$ and lager $W S_{2}(1-5 \mathrm{~kg}, D)$ The A-exciton response splits into two components from mono-layered (ML) and few-layered (FL) $W_{2}$. After the washing and transfer procedure, the monolayer content is even increased compared to the initial dispersion in SC. Peak shifts are attributed to solvatochromism. E-F) Photoluminescence-excitation contour plot of the 5-22k $\mathrm{g} \mathrm{WS}$ dispersion before (E) and after transfer to PMMA-THF-NMP $(F)$. In $F$, the $W S_{2} P L$ is completely masked by the solvent. 

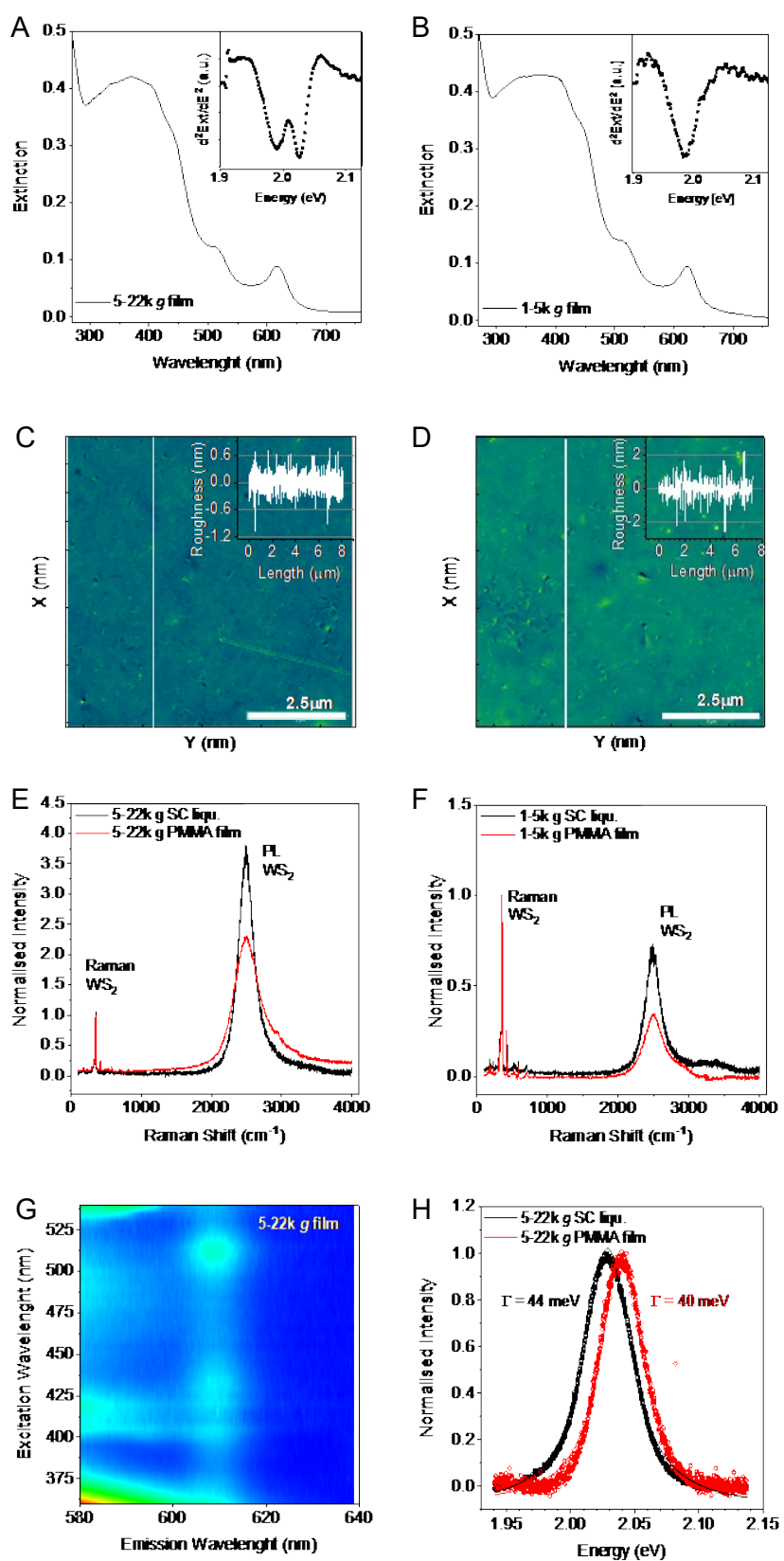

Figure 3: Characterisation of $W S_{2}-P M M A$ films. A-B) Extinction spectra of $W S_{2}-P M M A$ films produced containing small (5-22k $\mathrm{g}$, A) and larger (1-5k g, B) WS $\mathrm{S}_{2}$ nanosheets. Inset: second derivative of A-exciton showing the characteristic splitting of mono-layered and few-layered nanosheets for the 5-22k g sample confirming that no significant reaggregation occurred. $C$ D) AFM images of the $W S_{2}-P M M A$ film surface. Scale bar $2 \mu \mathrm{m}$. The surface roughness (inset) is in the $\mathrm{nm}$ range. C) $5-22 \mathrm{~kg}$, D) $1-5 \mathrm{~kg}$. E-F) Raman/PL spectra of the WS $\mathrm{S}_{2}$ PMMA films containing small $(5-22 k \mathrm{~g}, E)$ and larger $(1-5 k \mathrm{~g}, F) W S_{2}$ nanosheets compared to the asprepared dispersion in SC. The PL of the mono-layered $W S_{2}$ is well discernible in the film with minor shifts and broadening. G) Photoluminescence-excitation contour plot of the $W_{2}-P M M A$ film $(5-22 \mathrm{~kg})$ showing the monolayer $W_{2} P L$ at $\left.~ 610 \mathrm{~nm} . \mathrm{H}\right)$ Photoluminescence single spectrum (excitation $430 \mathrm{~nm})$ of $W_{2}(5-22 \mathrm{~kg})$ in aqueous $S C$ dispersion (black) and the $W_{2_{2}-}$ PMMA film (red). Solid lines are fits to single Lorentzians. 

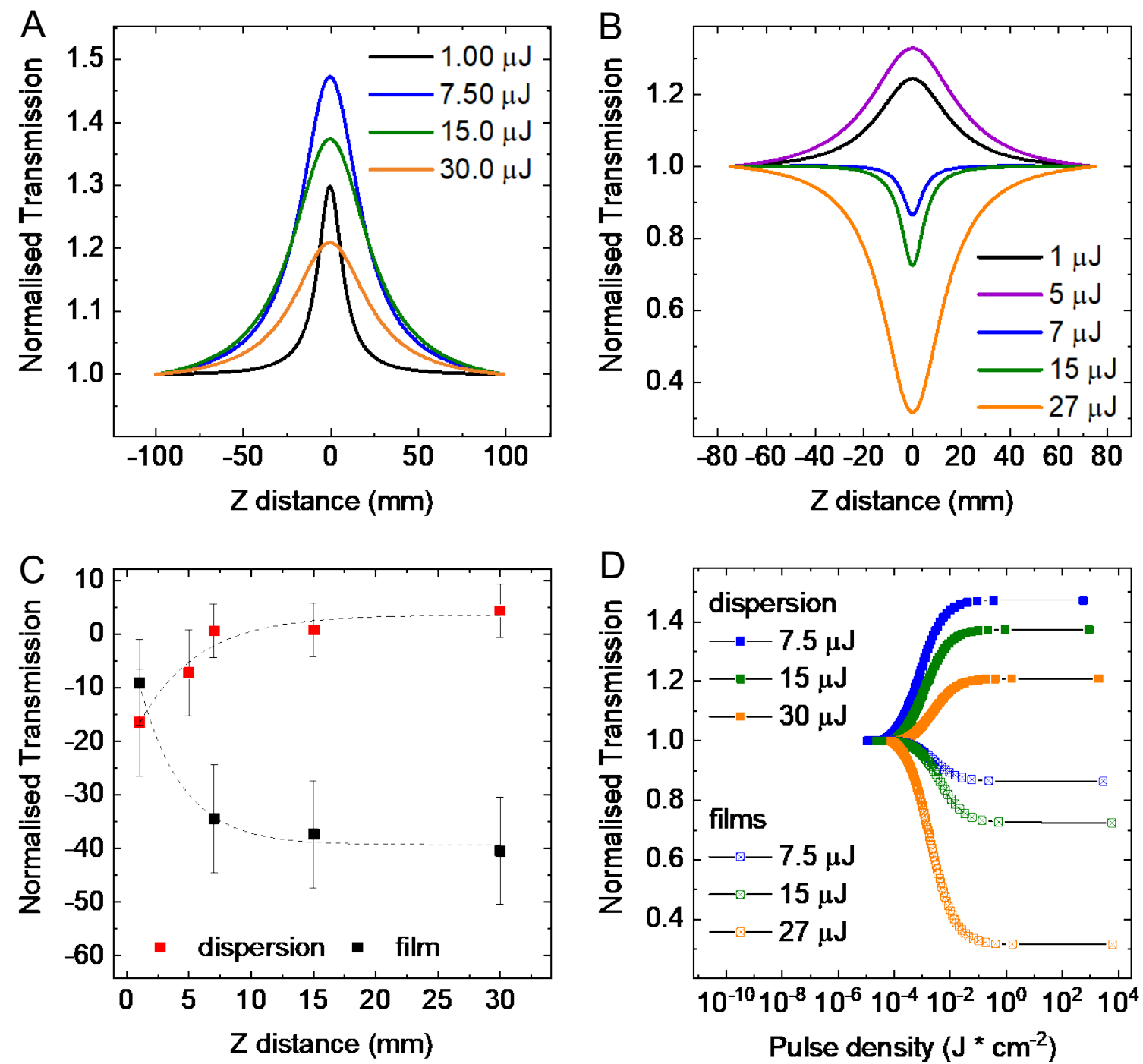

Figure 4: Figure. A-B) Open Aperture (OA) z-scan fitted data, normalised transmission vs. sample position (z) measured for variable incident energies A) $\mathrm{WS}_{2}-\mathrm{PMMA}$ film B) $\mathrm{WS}_{2}-\mathrm{H}_{2} \mathrm{O}$ $S C$ dispersion. C)Nonlinear optical coefficient dependence of energy input. D) NLO response of film and dispersion as a function of pulse fluence. 

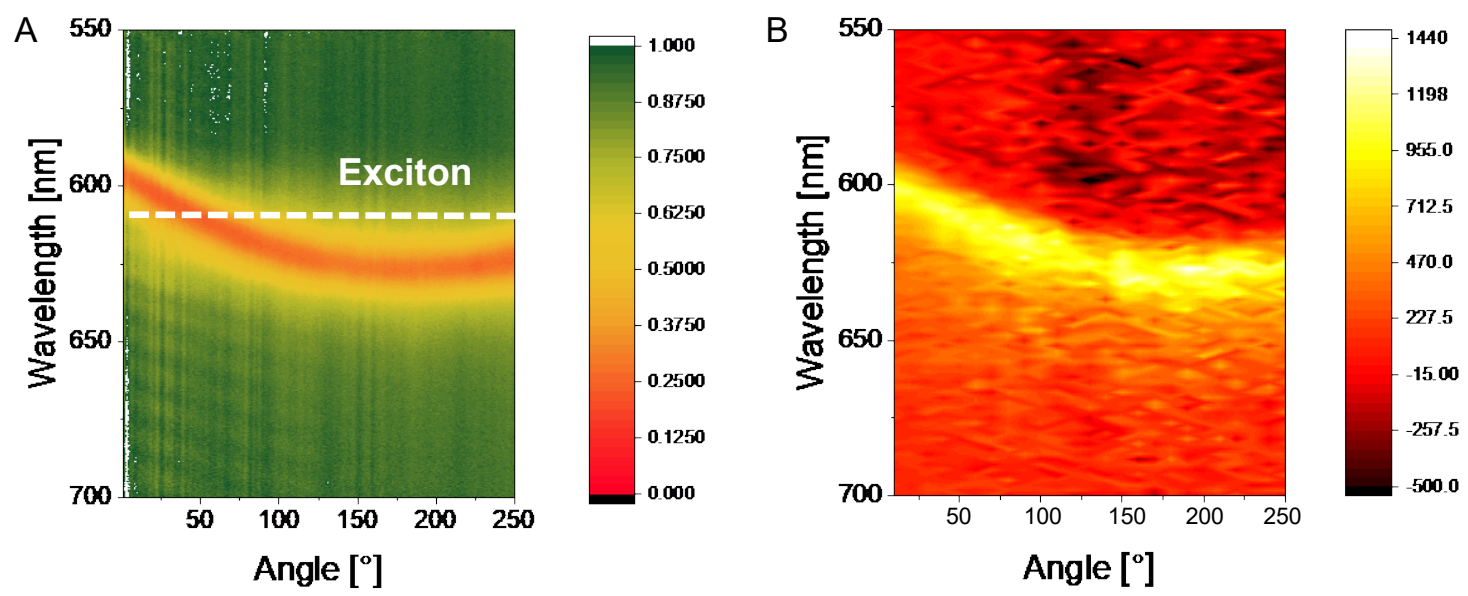

Figure 5: A) Angle-resolved reflectivity of a metal cavity containing a WS $\mathrm{S}_{2}-\mathrm{PMMA}$ layer. A cavity mode is formed crossing the $W_{2}$ exciton. The clear visibility of the mode confirms the homogeneity and low surface roughness of the layer. B) Angle-resolved PL spectra for the cavity in A. Emission occurs via the photonic cavity mode.

\section{Acknowledgement}

C.B. acknowledges the German research foundation DFG under Emmy-Noether grant BA4856/2-1. C.B., J.Z. and M.C.G. acknowledge the Volkswagen foundation under grant agreement $n^{\circ} 93404-93406$. W.J.B. gratefully acknowledges support by a research grant from Science Foundation Ireland (SFI) under Grant Number 12/IA/1306.

\section{Additional information}

Supplementary information is available.

\section{Competing financial interests}

The authors declare no competing financial interests. 


\section{References}

1. Jariwala, D.; Sangwan, V. K.; Lauhon, L. J.; Marks, T. J.; Hersam, M. C., Emerging Device Applications for Semiconducting Two-Dimensional Transition Metal Dichalcogenides. ACS Nano 2014, 8 (2), 1102-1120.

2. Mak, K. F.; Shan, J., Photonics and optoelectronics of 2D semiconductor transition metal dichalcogenides. Nat. Photon. 2016, 10 (4), 216-226.

3. Buscema, M.; Island, J. O.; Groenendijk, D. J.; Blanter, S. I.; Steele, G. A.; van der Zant, H. S. J.; Castellanos-Gomez, A., Photocurrent generation with two-dimensional van der Waals semiconductors. Chem. Soc. Rev. 2015, 44 (11), 3691-3718.

4. Voiry, D.; Yang, J.; Chhowalla, M., Recent Strategies for Improving the Catalytic Activity of 2D TMD Nanosheets Toward the Hydrogen Evolution Reaction. Adv. Mater. 2016, 28 (29), 61976206.

5. Low, T.; Chaves, A.; Caldwell, J. D.; Kumar, A.; Fang, N. X.; Avouris, P.; Heinz, T. F.; Guinea, F.; Martin-Moreno, L.; Koppens, F., Polaritons in layered two-dimensional materials. Nat. Mater. 2017, $16(2), 182-194$.

6. Li, B. L.; Wang, J.; Zou, H. L.; Garaj, S.; Lim, C. T.; Xie, J.; Li, N. B.; Leong, D. T., LowDimensional Transition Metal Dichalcogenide Nanostructures Based Sensors. Adv. Funct. Mater. 2016, 26 (39), 7034-7056.

7. Tao, H.; Gao, Y.; Talreja, N.; Guo, F.; Texter, J.; Sun, Z.; Yan, C., Two-Dimensional Nanosheets for Electrocatalysis in Energy Generation and Conversion. J. Mater. Chem. C. 2017, 16.

8. Pumera, M.; Sofer, Z.; Ambrosi, A., Layered transition metal dichalcogenides for electrochemical energy generation and storage. J. Mater. Chem. C. 2014, 2 (24), 8981-8987.

9. Mendoza-Sánchez, B.; Gogotsi, Y., Synthesis of Two-Dimensional Materials for Capacitive Energy Storage. Adv. Mater. 2016, 28 (29), 6104-6135.

10. Xiao, J.; Zhao, M.; Wang, Y.; Zhang, X., Excitons in atomically thin 2D semiconductors and their applications. Nanophotonics 2017, 6 (6), 1309.

11. Wang, G.; Chernikov, A.; Glazov, M. M.; Heinz, T. F.; Marie, X.; Amand, T.; Urbaszek, B., Excitons in atomically thin transition metal dichalcogenides. Rev. Mod. Phys. 2018, 90 (2), 021001.

12. Schneider, C.; Glazov, M. M.; Korn, T.; Höfling, S.; Urbaszek, B., Two-dimensional semiconductors in the regime of strong light-matter coupling. Nat. Commun. 2018, 9 (1), 2695.

13. You, J. W.; Bongu, S. R.; Bao, Q.; Panoiu, N. C., Nonlinear optical properties and applications of 2D materials: theoretical and experimental aspects. Nanophotonics 2018, $8(1), 63$.

14. Lemoine, K.; Zhang, L.; Dambournet, D.; Grenèche, J.-M.; Hémon-Ribaud, A.; Leblanc, M.; Borkiewicz, O. J.; Tarascon, J.-M.; Maisonneuve, V.; Lhoste, J., Synthesis by Thermal Decomposition of Two Iron Hydroxyfluorides: Structural Effects of Li Insertion. Chem. Mater. 2019, 31 (11), 4246-4257.

15. Yang, P.; Zou, X.; Zhang, Z.; Hong, M.; Shi, J.; Chen, S.; Shu, J.; Zhao, L.; Jiang, S.; Zhou, X.; Huan, Y.; Xie, C.; Gao, P.; Chen, Q.; Zhang, Q.; Liu, Z.; Zhang, Y., Batch production of 6inch uniform monolayer molybdenum disulfide catalyzed by sodium in glass. Nat. Commun. 2018, $9(1), 979$.

16. Zhao, Z.; Wu, D.; Guo, J.; Wu, E.; Jia, C.; Shi, Z.; Tian, Y.; Li, X.; Tian, Y., Synthesis of largearea 2D WS2 films and fabrication of a heterostructure for self-powered ultraviolet photodetection and imaging applications. J. Mater. Chem. C 2019, 7 (39), 12121-12126.

17. Harvey, A.; Boland, J. B.; Godwin, I.; Kelly, A. G.; Szydłowska, B. M.; Murtaza, G.; Thomas, A.; Lewis, D. J.; O’Brien, P.; Coleman, J. N., Exploring the versatility of liquid phase exfoliation: producing 2D nanosheets from talcum powder, cat litter and beach sand. 2D Mater. 2017, 4 (2).

18. Boland, J. B.; Harvey, A.; Tian, R.; Hanlon, D.; Vega-Mayoral, V.; Szydlowska, B.; Griffin, A.; Stimpel-Lindner, T.; Jaskaniec, S.; Nicolosi, V.; Duesberg, G.; Coleman, Jonathan N., Liquid phase exfoliation of $\mathrm{MoO} 2$ nanosheets for lithium ion battery applications. Nanoscale Adv. 2019, 1 (4), 1560-1570.

19. McAteer, D.; Godwin, I. J.; Ling, Z.; Harvey, A.; He, L.; Boland, C. S.; Vega-Mayoral, V.; Szydłowska, B.; Rovetta, A. A.; Backes, C.; Boland, J. B.; Chen, X.; Lyons, M. E. G.; Coleman, J. N., Liquid Exfoliated $\mathrm{Co}(\mathrm{OH}) 2$ Nanosheets as Low-Cost, Yet High-Performance, Catalysts for the Oxygen Evolution Reaction. Adv. En. Mater. 2018, 8 (15). 
20. Xie, S.; Istrate, O. M.; May, P.; Barwich, S.; Bell, A. P.; Khan, U.; Coleman, J. N., Boron nitride nanosheets as barrier enhancing fillers in melt processed composites. Nanoscale 2015, 7 (10), 4443 50.

21. Gebhardt, B.; Hof, F.; Backes, C.; Müller, M.; Plocke, T.; Thomsen, C.; Hauke, F.; Hirsch, A., Selective Reductive Sidewall Carboxylation of Semiconducting Single-Walled Carbon Nanotubes. J. Am. Chem. Soc. 2011, 133 (48), 19459-19473.

22. Vega-Mayoral, V.; Backes, C.; Hanlon, D.; Khan, U.; Gholamvand, Z.; O'Brien, M.; Duesberg, G. S.; Gadermaier, C.; Coleman, J. N., Photoluminescence from Liquid-Exfoliated WS2Monomers in Poly(Vinyl Alcohol) Polymer Composites. Adv. Func. Mater. 2016, 26 (7), 1028-1039.

23. Kang, J.; Sangwan, V. K.; Wood, J. D.; Hersam, M. C., Solution-Based Processing of Monodisperse Two-Dimensional Nanomaterials. Acc. Chem. Res. 2017, 50 (4), 943-951.

24. Backes, C.; Campi, D.; Szydlowska, B. M.; Synnatschke, K.; Ojala, E.; Rashvand, F.; Harvey, A.; Griffin, A.; Sofer, Z.; Marzari, N.; Coleman, J. N.; O'Regan, D. D., Equipartition of Energy Defines the Size-Thickness Relationship in Liquid-Exfoliated Nanosheets. ACS Nano 2019, 13 (6), 7050-7061.

25. Backes, C.; Szydłowska, B. M.; Harvey, A.; Yuan, S.; Vega-Mayoral, V.; Davies, B. R.; Zhao, P.-1.; Hanlon, D.; Santos, E. J. G.; Katsnelson, M. I.; Blau, W. J.; Gadermaier, C.; Coleman, J. N., Production of Highly Monolayer Enriched Dispersions of Liquid-Exfoliated Nanosheets by Liquid Cascade Centrifugation. ACS Nano 2016, 10 (1), 1589-1601.

26. Backes, C.; Smith, R. J.; McEvoy, N.; Berner, N. C.; McCloskey, D.; Nerl, H. C.; O’Neill, A.; King, P. J.; Higgins, T.; Hanlon, D.; Scheuschner, N.; Maultzsch, J.; Houben, L.; Duesberg, G. S.; Donegan, J. F.; Nicolosi, V.; Coleman, J. N., Edge and Confinement Effects Allow in situ Measurement of Size and Thickness of Liquid-Exfoliated Nanosheets. Nat. Commun. 2014, 5 , 4576.

27. Backes, C.; Paton, K. R.; Hanlon, D.; Yuan, S.; Katsnelson, M. I.; Houston, J.; Smith, R. J.; McCloskey, D.; Donegan, J. F.; Coleman, J. N., Spectroscopic metrics allow in situ measurement of mean size and thickness of liquid-exfoliated few-layer graphene nanosheets. Nanoscale 2016, 8 (7), 4311-4323.

28. Harvey, A.; Backes, C.; Boland, J. B.; He, X.; Griffin, A.; Szydlowska, B.; Gabbett, C.; Donegan, J. F.; Coleman, J. N., Non-resonant light scattering in dispersions of 2D nanosheets. Nat. Commun. 2018, 9 (1), 4553.

29. Yadgarov, L.; Choi, C. L.; Sedova, A.; Cohen, A.; Rosentsveig, R.; Bar-Elli, O.; Oron, D.; Dai, H.; Tenne, R., Dependence of the Absorption and Optical Surface Plasmon Scattering of MoS2 Nanoparticles on Aspect Ratio, Size, and Media. ACS Nano 2014, 8 (4), 3575-3583.

30. Saakov, V. S.; Drapkin, V. Z.; Krivchenko, A. I.; Rozengart, E. V.; Bogachev, Y. V.; Knyazev, M. N., Derivative Spectrophotometry and Electron Spin Resonance (ESR) Spectroscopy for Ecological and Biological Questions. Springer-Verlag Wien: 2013.

31. Gutierrez, H. R.; Perea-Lopez, N.; Elias, A. L.; Berkdemir, A.; Wang, B.; Lv, R.; Lopez-Urias, F.; Crespi, V. H.; Terrones, H.; Terrones, M., Extraordinary room-temperature photoluminescence in triangular WS2 monolayers. Nano Lett. 2013, 13 (8), 3447-54.

32. Backes, C.; Szydlowska, B. M.; Harvey, A.; Yuan, S.; Vega-Mayoral, V.; Davies, B. R.; Zhao, P. L.; Hanlon, D.; Santos, E. J.; Katsnelson, M. I.; Blau, W. J.; Gadermaier, C.; Coleman, J. N., Production of Highly Monolayer Enriched Dispersions of Liquid-Exfoliated Nanosheets by Liquid Cascade Centrifugation. ACS Nano 2016, 10 (1), 1589-601.

33. Ajayi, O. A.; Ardelean, J. V.; Shepard, G. D.; Wang, J.; Antony, A.; Taniguchi, T.; Watanabe, K.; Heinz, T. F.; Strauf, S.; Zhu, X. Y.; Hone, J. C., Approaching the intrinsic photoluminescence linewidth in transition metal dichalcogenide monolayers. 2D Mater. 2017, 4 (3).

34. Reale, F.; Palczynski, P.; Amit, I.; Jones, G. F.; Mehew, J. D.; Bacon, A.; Ni, N.; Sherrell, P. C.; Agnoli, S.; Craciun, M. F.; Russo, S.; Mattevi, C., High-Mobility and High-Optical Quality Atomically Thin WS2. Sci Rep 2017, 7 (1), 14911.

35. Ogilvie, S. P.; Large, M. J.; Fratta, G.; Meloni, M.; Canton-Vitoria, R.; Tagmatarchis, N.; Massuyeau, F.; Ewels, C. P.; King, A. A. K.; Dalton, A. B., Considerations for spectroscopy of liquid-exfoliated 2D materials: emerging photoluminescence of N-methyl-2-pyrrolidone. Sci. Rep. 2017, 7 (1), 16706. 
36. Raja, A.; Waldecker, L.; Zipfel, J.; Cho, Y.; Brem, S.; Ziegler, J. D.; Kulig, M.; Taniguchi, T.; Watanabe, K.; Malic, E.; Heinz, T. F.; Berkelbach, T. C.; Chernikov, A., Dielectric disorder in two-dimensional materials. Nat. Nanotechnol. 2019, 14 (9), 832-837.

37. Ma, C.; Yan, J.; Huang, Y.; Yang, G., Photoluminescence manipulation of WS2 flakes by an individual Si nanoparticle. Mater. Horizons 2019, 6 (1), 97-106.

38. Feng, M.; Zhan, H.; Chen, Y., Nonlinear optical and optical limiting properties of graphene families. Appl. Phys. Lett. 2010, 96 (3).

39. Zhang, J.; Ouyang, H.; Zheng, X.; You, J.; Chen, R.; Zhou, T.; Sui, Y.; Liu, Y.; Cheng, X.; Jiang, T., Ultrafast saturable absorption of MoS2 nanosheets under different pulse-width excitation conditions. Opt Lett 2018, 43 (2), 243-246.

40. Zhou, K. G.; Zhao, M.; Chang, M. J.; Wang, Q.; Wu, X. Z.; Song, Y.; Zhang, H. L., Sizedependent nonlinear optical properties of atomically thin transition metal dichalcogenide nanosheets. Small 2015, 11 (6), 694-701.

41. Szydłowska, B. M.; Tywoniuk, B.; Blau, W. J., Size-Dependent Nonlinear Optical Response of Black Phosphorus Liquid Phase Exfoliated Nanosheets in Nanosecond Regime. ACS Photonics 2018, 5 (9), 3608-3612.

42. Backes, C.; Englert, J.; Bernhard, N.; Hauke, F.; Hirsch, A., Optical Visualization of Carbon Nanotubes-a Unifying Linkage Between Microscopic and Spectroscopic Characterization Techniques. Small 2010, 6 (18), 1968-1973.

43. Low, T.; Chaves, A.; Caldwell, J. D.; Kumar, A.; Fang, N. X.; Avouris, P.; Heinz, T. F.; Guinea, F.; Martin-Moreno, L.; Koppens, F., Polaritons in layered two-dimensional materials. Nat. Mater. 2017, $16(2), 182-194$.

44. Liu, X.; Galfsky, T.; Sun, Z.; Xia, F.; Lin, E.-c.; Lee, Y.-H.; Kéna-Cohen, S.; Menon, V. M., Strong light-matter coupling in two-dimensional atomic crystals. Nat. Photonics 2014, 9, 30.

45. Wu, S.; Buckley, S.; Jones, A. M.; Ross, J. S.; Ghimire, N. J.; Yan, J.; Mandrus, D. G.; Yao, W.; Hatami, F.; Vučković, J.; Majumdar, A.; Xu, X., Control of two-dimensional excitonic light emission via photonic crystal. 2D Mater. 2014, 1 (1), 011001.

46. Janisch, C.; Song, H.; Zhou, C.; Lin, Z.; Elías, A. L.; Ji, D.; Terrones, M.; Gan, Q.; Liu, Z., MoS2monolayers on nanocavities: enhancement in light-matter interaction. 2D Mater. 2016, 3 (2), 025017 .

47. Graf, A.; Tropf, L.; Zakharko, Y.; Zaumseil, J.; Gather, M. C., Near-infrared exciton-polaritons in strongly coupled single-walled carbon nanotube microcavities. Nat. Commun. 2016, 7, 13078.

48. Graf, A.; Held, M.; Zakharko, Y.; Tropf, L.; Gather, M. C.; Zaumseil, J., Electrical pumping and tuning of exciton-polaritons in carbon nanotube microcavities. Nat. Mater. 2017, 16 (9), 911-917. 